OCHA-PP-151

AJC-HEP-33

\title{
Production of CP-even and CP-odd Higgs bosons at Muon colliders
}

\author{
Eri Asakawa*, Akio Sugamoto*, and Isamu Watanabe** \\ * Department of Physics and \\ Graduate School of Humanities and Sciences, Ochanomizu University \\ Tokyo 112-8610, Japan \\ ** Akita Keizaihoka University \\ Akita 010-8515, Japan
}

\begin{abstract}
In the $s$-channel Higgs-boson-exchange processes, the interference between the amplitudes for CP-even and CP-odd Higgs bosons is sizable, if the helicities of initial and final particles are properly fixed and if the mass difference between these bosons is not much larger than their decay widths. We discuss this interference effect in the process $\mu^{+} \mu^{-} \rightarrow t \bar{t}$. Examining the effects gives us information on CP-parity for Higgs bosons and on the sign of a product of the coupling constants for $H \mu^{-} \mu^{+}$, $H t \bar{t}, A \mu^{-} \mu^{+}$and $A t \bar{t}$ vertices. The feasibility of observing the interference effect in future muon colliders is evaluated in the framework of the minimal supersymmetric extension of the standard model as an example.
\end{abstract}




\section{Introduction}

The standard model (SM) of particle physics predicts one physical neutral CP-even Higgs boson. It can be extended by increasing Higgs fields. In models with more than one Higgs doublets there are extra two neutral and two charged physical Higgs bosons for each additional doublet. If $\mathrm{CP}$ is a good symmetry, one neutral boson is CP-even $(H)$ and the other is CP-odd $(A)$. Searches for the Higgs bosons and precise measurements of their properties are indispensable for understanding the mechanism of electroweak symmetry breaking and knowing which model is realized in nature.

A muon collider is one of the ideal machines to look for the Higgs bosons [1, 2], where $\mu^{+} \mu^{-}$pairs are directly annihilated into $H$ and $A$ bosons. The muon beams can be polarized [3]. The feasibility of detecting the Higgs bosons and measuring their properties, such as masses, total widths and decay branching fractions has been studied [1, 2, 4, 5, 6, 7]. They have covered the special cases where $H$ and $A$ bosons are distributed overlapping their resonances [四] which are often expected in the minimal supersymmetric extension of the SM (MSSM) and where mixing between $H$ and $A$ bosons occurs due to loop-induced CP violation [5]. Moreover, determining the CP-nature of Higgs bosons has been also discussed by using initial muon polarizations [6].

We here take into consideration of overlapped resonances, especially concentrating on interference between the resonances. As was pointed out recently in Ref. [8] where the process $\gamma \gamma \rightarrow t \bar{t}$ is studied, the amplitudes of $s$-channel exchanges for $H$ and $A$ bosons can sizably interfere with each other, if the helicities of the initial and final particles are fixed properly and if the mass difference between these Higgs bosons is at most of the same order as their decay widths. This interference effect disappears if the helicities of both initial and

final particles are not fixed. Similar effects are also expected in the process $\mu^{+} \mu^{-} \rightarrow t \bar{t}$.

In this paper, we discuss the interference effects on the cross section of the process $\mu^{+} \mu^{-} \rightarrow t \bar{t}$ assuming definite helicities of the initial and final particles. Although the 
process $\mu^{+} \mu^{-} \rightarrow t \bar{t}$ is also generated by $s$-channel $\gamma$ - and $Z$-exchange diagrams, their contributions are small under the helicity combinations which induce the $s$-channel $H$ - and $A$-exchange amplitudes. The interference effects are measured by the difference between the cross sections for various helicity selections. We estimate an asymmetry between these cross sections. Examining the effects gives us information on CP-parity for the Higgs bosons. In addition, the asymmetry provides information about the sign of a product of the coupling constants for $H \mu^{-} \mu^{+}, H t \bar{t}, A \mu^{-} \mu^{+}$and $A t \bar{t}$ vertices. Since this sign depends on a model, we can judge a type of the model from a viewpoint of the coupling constants. An example is given in the MSSM which contains two Higgs doublets.

This paper is organized as follows. In Sect. 2 we obtain helicity amplitudes of the process $\mu^{+} \mu^{-} \rightarrow t \bar{t}$. In Sect. 3 interference effects are discussed and an asymmetry between the cross sections is defined. In Sect. 4 numerical estimates of the cross sections and the asymmetry are given. The degree of polarization of muon beams and a method of helicity observation of final top pairs are also considered. We give conclusions in the last section.

\section{Helicity Amplitudes}

The process $\mu^{+} \mu^{-} \rightarrow t \bar{t}$ receives contributions from the diagrams in which $H, A, \gamma$ and $Z$ are exchanged as shown in Fig. 1. We express the helicity amplitudes for these diagrams as

$$
\mathcal{M}_{H}^{\Lambda \bar{\Lambda} \lambda \bar{\lambda}}, \mathcal{M}_{A}^{\Lambda \bar{\Lambda} \lambda \bar{\lambda}}, \mathcal{M}_{\gamma}^{\Lambda \bar{\Lambda} \lambda \bar{\lambda}}, \mathcal{M}_{Z}^{\Lambda \bar{\Lambda} \lambda \bar{\lambda}}
$$

The superscripts $\Lambda$ and $\bar{\Lambda}$ denote the initial $\mu^{-}$and $\mu^{+}$helicities, while $\lambda$ and $\bar{\lambda}$ the final $t$ and $\bar{t}$ helicities in the center-of-mass frame. Defining $\lambda_{i}, \lambda_{f}$ as $\Lambda-\bar{\Lambda}, \lambda-\bar{\lambda}$, respectively, the amplitudes are given by

$$
\begin{aligned}
\mathcal{M}_{H}^{\Lambda \bar{\Lambda} \lambda \bar{\lambda}} & =-4 \Lambda \lambda a_{\mu} a_{t} g^{2} \frac{m_{\mu} m_{t}}{m_{W}^{2}} \beta_{\mu} \beta_{t} \frac{s}{s-m_{H}^{2}+i m_{H} \Gamma_{H}} \delta_{\lambda_{i} 0} \delta_{\lambda_{f} 0} \\
\mathcal{M}_{A}^{\Lambda \bar{\Lambda} \lambda \bar{\lambda}} & =-b_{\mu} b_{t} g^{2} \frac{m_{\mu} m_{t}}{m_{W}^{2}} \frac{s}{s-m_{A}^{2}+i m_{A} \Gamma_{A}} \delta_{\lambda_{i} 0} \delta_{\lambda_{f} 0} \\
\mathcal{M}_{\gamma}^{\Lambda \bar{\Lambda} \lambda \bar{\lambda}} & =\bar{\Lambda} \bar{\lambda} \frac{16}{3} \pi \alpha_{Q E D} K_{\mu} K_{t} d_{\lambda_{i} \lambda_{f}}^{1}
\end{aligned}
$$




$$
\begin{aligned}
\mathcal{M}_{Z}^{\Lambda \bar{\Lambda} \lambda \bar{\lambda}} & =\frac{\pi \alpha_{Q E D}}{2 \sin ^{2} \theta_{W} \cos ^{2} \theta_{W}} K_{\mu} K_{t}\left[F_{0} \delta_{\lambda_{i} 0} \delta_{\lambda_{f} 0}-\bar{\Lambda} \bar{\lambda} F_{\mu} F_{t} d_{\lambda_{i} \lambda_{f}}^{1}\right] \frac{s}{s-m_{Z}^{2}}, \\
K_{\mu} & =\delta_{\lambda_{i} 0} \sqrt{\frac{2}{s}} m_{\mu}+\left|\lambda_{i}\right|, \quad K_{t}=\delta_{\lambda_{f} 0} \sqrt{\frac{2}{s} m_{t}+\left|\lambda_{f}\right|,} \\
F_{0} & =\frac{s-m_{Z}^{2}}{m_{Z}^{2}} \\
F_{\mu} & =-1+4 \sin ^{2} \theta_{W}+\lambda_{i} \beta_{\mu}, \quad F_{t}=+1-\frac{8}{3} \sin ^{2} \theta_{W}-\lambda_{f} \beta_{t},
\end{aligned}
$$

where $g$ is the weak coupling constant; $m_{\mu}, m_{t}, m_{W}$ and $m_{Z}$ are the masses of muon, top quark, $W$ boson and $Z$ boson; $\beta_{t}$ and $\beta_{\mu}$ are the velocities of the top quarks and the muons in the center-of-mass frame; $s$ is the collision energy-squared; and $d$ is the Wigner's $d$ function. The masses and the total decay widths of Higgs bosons are denoted by $m_{H, A}$ and $\Gamma_{H, A}$. The coefficients $a_{\mu}, a_{t}, b_{\mu}$ and $b_{t}$ are the coupling constants for $H \mu^{-} \mu^{+}, H t \bar{t}$, $A \mu^{-} \mu^{+}$and $A t \bar{t}$ vertices, respectively. In the framework of the MSSM, they are expressed as

$$
a_{\mu}=-\frac{1}{2} \frac{\cos \alpha}{\cos \beta}, \quad a_{t}=-\frac{1}{2} \frac{\sin \alpha}{\sin \beta}, \quad b_{\mu}=\frac{1}{2} \tan \beta, \quad b_{t}=\frac{1}{2} \cot \beta
$$

Here $\alpha$ is the mixing angle of the two CP-even Higgs bosons and $\tan \beta$ is the ratio of the vacuum expectation values of two Higgs doublets. Here $-4 a_{\mu} a_{t}$ is almost unity within the accuracy of $13 \%$ for $\tan \beta=1-100$ in the MSSM, which is comparable with $4 b_{\mu} b_{t}=1$.

The Higgs-exchange diagrams can contribute only for $\lambda_{i}=\lambda_{f}=0$. The absolute values of $\mathcal{M}_{\gamma}$ and $\mathcal{M}_{Z}$ in this case are proportional to $m_{\mu}$, and negligibly small around the mass poles of the Higgs bosons if the masses of the Higgs bosons are far above $m_{Z}$, as will be seen in the subsection 3.3 .

\section{$3 \quad$ Interference Effects}

The signs of Higgs-exchange amplitudes change under CP transformation. Expressing $\mathcal{M}_{H, A}^{R R R R}$ as $\mathcal{M}_{H, A}$, where $R(L)$ means the helicity $+1 / 2(-1 / 2)$, the helicity dependence of the Higgs-exchange amplitudes is summarized in Table 1. With $|R R\rangle$ and $|L L\rangle$ being 
spin-zero states of $t_{R} \bar{t}_{R}$ or $\mu_{R}^{+} \mu_{R}^{-}$, and $t_{L} \bar{t}_{L}$ or $\mu_{L}^{+} \mu_{L}^{-}$system, respectively, these states are interchanged under CP transformation,

$$
\begin{aligned}
\mathcal{C P}|R R\rangle & =-|L L\rangle, \\
\mathcal{C P}|L L\rangle & =-|R R\rangle .
\end{aligned}
$$

For example, $\mathcal{M}_{H}^{R R R R}$ and $\mathcal{M}_{H}^{R R L L}$ are related by $\mathrm{CP}$ transformation of the final $t \bar{t}$ system and have the same absolute value of the amplitudes with the different signs. As for $\mathcal{M}_{A}$, the odd contribution of CP transformation for the final $t \bar{t}$ state is cancelled by the transformation of the CP-odd Higgs boson.

With the helicities fixed, the interference term of the amplitudes for the Higgs bosons, $\Delta \sigma^{\Lambda \bar{\Lambda} \lambda \bar{\lambda}}$, is given by

$$
\Delta \sigma^{\Lambda \bar{\Lambda} \lambda \bar{\lambda}}=\frac{N_{c}}{8 \pi s} \frac{\beta_{t}}{\beta_{\mu}} \mathcal{R} e\left[\mathcal{M}_{\phi_{1}}^{\Lambda \bar{\Lambda} \lambda \bar{\lambda}} \cdot \mathcal{M}_{\phi_{2}}^{* \Lambda \bar{\Lambda} \lambda \bar{\lambda}}\right]
$$

where $N_{c}$ is the color factor of the top quark, $\phi_{1}$ and $\phi_{2}$ denote the relevant two Higgs bosons. The interference between the amplitudes for two Higgs bosons is manifestly dependent on the relative CP-parity of the Higgs bosons. According to Tabel 1 , the terms $\Delta \sigma^{\Lambda \bar{\Lambda} \lambda \bar{\lambda}}$ for different helicity states are related to each other. For $\phi_{1}=H$ and $\phi_{2}=A$,

$$
\Delta \sigma^{R R R R}=\Delta \sigma^{L L L L}=-\Delta \sigma^{R R L L}=-\Delta \sigma^{L L R R}
$$

while for $\phi_{1}=\phi_{2}=H$ or $\phi_{1}=\phi_{2}=A$

$$
\Delta \sigma^{R R R R}=\Delta \sigma^{L L L L}=\Delta \sigma^{R R L L}=\Delta \sigma^{L L R R}
$$

Therefore, the comparison of the interference terms between the different helicity states could provide a useful information on the Higgs CP-parity.

As an index for the interference term between two Higgs bosons, we define an asymmetry of the cross sections as

$$
\mathcal{A} \equiv \frac{\sigma^{R R R R}+\sigma^{L L L L}-\sigma^{R R L L}-\sigma^{L L R R}}{\sigma^{R R R R}+\sigma^{L L L L}+\sigma^{R R L L}+\sigma^{L L R R}} .
$$


where $\sigma^{\Lambda \bar{\Lambda} \lambda \bar{\lambda}}$ denotes the cross section of $\mu^{+} \mu^{-} \rightarrow t \bar{t}$ with fixed helicities which is given by

$$
\sigma^{\Lambda \bar{\Lambda} \lambda \bar{\lambda}}=\frac{N_{c}}{32 \pi s} \frac{\beta_{t}}{\beta_{\mu}} \int_{-1}^{+1} d \cos \theta\left|\mathcal{M}^{\Lambda \bar{\Lambda} \lambda \bar{\lambda}}\right|^{2},
$$

with $\mathcal{M}^{\Lambda \bar{\Lambda} \lambda \bar{\lambda}}$ denoting the sum of relevant amplitudes. $\theta$ is the scattering angle of the top quarks in center-of-mass frame. If the cross section receives contributions dominantly from the diagrams mediated by the two Higgs bosons, the cross section can be written as

$$
\sigma^{\Lambda \bar{\Lambda} \lambda \bar{\lambda}}=\frac{N_{c}}{16 \pi s} \frac{\beta_{t}}{\beta_{\mu}}\left\{\left|\mathcal{M}_{\phi_{1}}\right|^{2}+\left|\mathcal{M}_{\phi_{2}}\right|^{2}\right\}+\Delta \sigma^{\Lambda \bar{\Lambda} \lambda \bar{\lambda}}
$$

and the asymmetry becomes

$$
\begin{aligned}
\mathcal{A} & =\frac{2 \mathcal{R} e\left[\mathcal{M}_{H} \cdot \mathcal{M}_{A}^{*}\right]}{\left|\mathcal{M}_{H}\right|^{2}+\left|\mathcal{M}_{A}\right|^{2}} & \left(\phi_{1}=H \text { and } \phi_{2}=A\right), \\
& =0 & \left(\phi_{1}=\phi_{2}=H \text { or } \phi_{1}=\phi_{2}=A\right) .
\end{aligned}
$$

Therefore, observation of a non-vanishing value for $\mathcal{A}$ indicates that the two Higgs bosons have different CP-parities.

In addition, from the sign of $\mathcal{A}$, we can also learn the sign of the product of the coupling constants for $H \mu^{-} \mu^{+}, H t \bar{t}, A \mu^{-} \mu^{+}$and $A t \bar{t}$ vertices. From eq. (2.2) and eq. (2.3),

$$
\mathcal{R} e\left[\mathcal{M}_{H} \cdot \mathcal{M}_{A}^{*}\right]=\left(a_{\mu} \cdot a_{t} \cdot b_{\mu} \cdot b_{t}\right) \beta_{\mu} \beta_{t} g^{4} \frac{m_{\mu}^{2} m_{t}^{2}}{m_{W}^{4}} s^{2} \mathcal{D}
$$

where

$$
\mathcal{D} \equiv \frac{\left(s-m_{H}^{2}\right)\left(s-m_{A}^{2}\right)+m_{H} m_{A} \Gamma_{H} \Gamma_{A}}{\left[\left(s-m_{H}^{2}\right)^{2}+m_{H}^{2} \Gamma_{H}^{2}\right]\left[\left(s-m_{A}^{2}\right)^{2}+m_{A}^{2} \Gamma_{A}^{2}\right]} .
$$

The sign of $\mathcal{A}$ is coincident with the sign of $\left(a_{\mu} \cdot a_{t} \cdot b_{\mu} \cdot b_{t}\right)$ in the region of $\mathcal{D} \geq 0$ and is opposite to in the region of $\mathcal{D}<0$.

\section{Numerical Estimates}

\subsection{Interference effect in MSSM}

Assuming the MSSM as an example for various multi-Higgs-doublet models, we present numerical estimates of the asymmetry $\mathcal{A}$. The MSSM include three neutral Higgs bosons, 
two of which are CP-even and the other is CP-odd. The Higgs sector can be parameterized by two parameters, the mass of the CP-odd Higgs boson $m_{A}$ and the ratio of the vacuum expectation values $\tan \beta$. If $m_{A}$ is sufficiently large, the mass of the heavier CP-even Higgs boson $m_{H}$ becomes approximately degenerated into $m_{A}$. For definiteness, we take $m_{A}=400 \mathrm{GeV}$ and $\tan \beta=3,7,15$ and 30 . The masses, the decay widths and the decay branching ratios of the Higgs bosons from which we derive the coupling constants among Higgs and fermions are computed by the program HDECAY [9], which are listed in Table 2. For the input parameters in the program, the sfermion mass scale is set for $m_{\mathrm{SusY}}=1 \mathrm{TeV}$, the $\mathrm{SU}(2)$ gaugino mass parameter $M_{2}$ for $500 \mathrm{GeV}$ and the higgsino mixing mass parameter $\mu$ for $-500 \mathrm{GeV}$, which result in heavy supersymmetric particles. No new particles other than Higgs bosons are produced by the decay of the $H$ and $A$ bosons for the parameters.

In Fig. 2 we show the center-of-mass-energy dependence of the cross sections for the helicity combinations satisfying $\lambda_{i}=\lambda_{f}=0$. The cross sections for $\Lambda=\bar{\Lambda}=\lambda=\bar{\lambda}$, $\sigma^{L L L L}$ and $\sigma^{R R R R}$, are different from those for $\Lambda=\bar{\Lambda} \neq \lambda=\bar{\lambda}, \sigma^{L L R R}$ and $\sigma^{R R L L}$. These differences come from the interference effect between the Higgs-exchange amplitudes. The peak cross section is maximized at $\tan \beta \sim 7$, as $\sigma \simeq 4000 \mathrm{fb}$. The cross section mediated by $\gamma$ - and $Z$-exchange diagrams are smaller than $0.01 \mathrm{fb}$ for $\lambda_{i}=\lambda_{f}=0$, and generally negligible in these energy range. A Large interference effect can be seen when the mass difference between $H$ and $A$ bosons is smaller than their widths and two Higgs-exchange amplitudes have comparable magnitudes, as is shown for $\tan \beta=15$ and 30 in Fig. 2.

The asymmetry $\mathcal{A}$ for the cross sections is given in Table 3 for several values of $\sqrt{s}$ at and around the resonances. The asymmetry reaches to $\mathcal{O}(1)$ in certain ranges of $\sqrt{s}$, showing the strong effect of the interference between $H$ and $A$. Since $\mathcal{A}$ is negative for $\sqrt{s}<m_{H, A}$, we can deduce

$$
a_{\mu} \cdot a_{t} \cdot b_{\mu} \cdot b_{t}<0
$$

which is consistent with eq. (2.8). 


\subsection{Beam polarization and helicity observation}

The above arguments did not take account of the degree of polarization of the initial muon beams and the helicity observation of the final top pairs. As was mentioned in section 2, the absolute values of the amplitudes of $\gamma$ - and $Z$-exchange diagrams with helicity combinations other than $\lambda_{i}=\lambda_{f}=0$ are generally larger than those with $\lambda_{i}=\lambda_{f}=0$ (Fig. 3). Therefore, the background processes $\mu^{+} \mu^{-} \rightarrow \gamma / Z \rightarrow t \bar{t}$ should be considered as long as the polarization of muon beams and the efficiency of helicity observation of top pairs are not perfect. The cross sections are contaminated with these backgrounds.

There are some statistical methods to measure the top-quark helicity [8, 10, 11]. As an illustration, we follow Ref. [8]. The bottom quark decaying from a top quark has the angular distribution proportional to $0.5-0.2 \lambda \cos \theta$, where $\theta$ is the emission angle of the bottom quark in the rest frame of the decaying top quark with respect to the direction of the top momentum in the $t \bar{t}$ c.m. frame. The anti-bottom quark has the distributon proportional to $0.5+0.2 \bar{\lambda} \cos \bar{\theta}$, where $\bar{\theta}$ is the emission angle of the anti-bottom quark Đ. Therefore, an event with the top quark decaying forward and with the anti-top quark decaying backward is most likely to be a $t_{L} \bar{t}_{L}$ event. Applying the emission angle cuts $F$ (forward, $0<\cos \theta, \cos \bar{\theta}<+1$ ) and $B$ (backward, $-1<\cos \theta, \cos \bar{\theta}<0$ ) to the bottom quark diretion, the counted numbers of polarized top and anti-top decay events are reduced by the factor $R$ and $\bar{R}$, respectively.

$$
\begin{aligned}
& R(F, \lambda)=\frac{1-2 \lambda r}{2}, \quad R(B, \lambda)=\frac{1+2 \lambda r}{2} \\
& \bar{R}(F, \bar{\lambda})=\frac{1+2 \bar{\lambda} r}{2}, \quad \bar{R}(B, \bar{\lambda})=\frac{1-2 \bar{\lambda} r}{2}
\end{aligned}
$$

where $r$ is $r_{B}-r_{F}$ in Ref. [8], and its value is estimated to be 0.2 in this method. If one observe the direction of the charged lepton from the $t \rightarrow b W \rightarrow b l \nu$ decay [11], instead of the bottom quark direction, $r$ reaches to be 0.5 in the Born approximation.

\footnotetext{
${ }^{1}$ In order to derive the emission angles, $\theta$ and $\bar{\theta}$, one needs to identify the charge and the momentum of the top and the anti-top quarks. The tasks can be done by observing their decay products. For example, when one $W$ decays leptonically and the other decays hadronically, we can generally reconstruct the top and the anti-top decay kinematics. Semileptonic decays of the $B$ mesons can be used when both $W$ 's decay hadronically. The efficiency of the analysis of such $t \bar{t}$ reconstructions is discussed in Ref. [12].
} 
We define the effective cross sections as follows:

$$
\sigma\left(P_{\mu^{-}}, P_{\mu^{+}}, c, \bar{c}\right)=\sum_{\Lambda \bar{\Lambda} \lambda \bar{\lambda}} \sigma^{\Lambda \bar{\Lambda} \lambda \bar{\lambda}} \frac{\left(1+2 \Lambda P_{\mu^{-}}\right)}{2} \frac{\left(1+2 \bar{\Lambda} P_{\mu^{+}}\right)}{2} R(c, \lambda) \bar{R}(\bar{c}, \bar{\lambda}),
$$

where $P_{\mu^{-}}$and $P_{\mu^{+}}$denote the degree of polarizations of $\mu^{-}$and $\mu^{+}$, and $c$ and $\bar{c}$ are $F$ or $B$, respectively. The effective cross sections are shown in Fig. 团, assuming $\left|P_{\mu^{-}}\right|=\left|P_{\mu^{+}}\right|=0.6$ as an example. To extract the Higgs signal efficiently, we should select as $P_{\mu^{-}}=P_{\mu^{+}}=P$ and, $c=F$ and $\bar{c}=B$ or $c=B$ and $\bar{c}=F$. In these selections, the background cross sections of $\gamma$ and $Z$ are independent on the signs of $P$ 's and the choice of $c$. There is another interference contribution between the Higgs-exchange and the $\gamma$ - or $Z$-exchange amplitude for $\lambda_{i}=\lambda_{f}=0$, and this interference has helicity dependence, however, it is negligibly small. With the polarizations and the emission angle cuts, we can define another asymmetry of the cross sections between the different cuts, with $P$ unchanged.

$$
\begin{gathered}
\mathcal{A}^{\prime}=\frac{\sigma(P, P, B, F)-\sigma(P, P, F, B)}{\sigma(P, P, B, F)+\sigma(P, P, F, B)} \\
=\frac{\sigma^{d i f f}}{\sigma^{\text {tot }}} \\
\sigma^{d i f f}=\frac{\epsilon}{4}\left\{(1+P)^{2}\left(\sigma^{R R R R}-\sigma^{R R L L}\right)+(1-P)^{2}\left(\sigma^{L L R R}-\sigma^{L L L L}\right)\right\} \\
\sigma^{t o t}=\frac{1}{8}\left\{(1+P)^{2}\left[\left(1+\epsilon^{2}\right)\left(\sigma^{R R R R}+\sigma^{R R L L}\right)+\left(1-\epsilon^{2}\right)\left(\sigma^{R R R L}+\sigma^{R R L R}\right)\right]\right. \\
+\quad\left(1-P^{2}\right)\left[\left(1+\epsilon^{2}\right)\left(\sigma^{L R R R}+\sigma^{R L L L}+\sigma^{R L R R}+\sigma^{L R L L}\right)\right. \\
\left.+\left(1-\epsilon^{2}\right)\left(\sigma^{L R R L}+\sigma^{R L L R}+\sigma^{R L R L}+\sigma^{L R L R}\right)\right] \\
\left.+\quad(1-P)^{2}\left[\left(1+\epsilon^{2}\right)\left(\sigma^{L L R R}+\sigma^{L L L L}\right)+\left(1-\epsilon^{2}\right)\left(\sigma^{L L R L}+\sigma^{L L L R}\right)\right]\right\}
\end{gathered}
$$

As is demonstrated in Table 3, the ratio of the asymmetries $\mathcal{A} / \mathcal{A}^{\prime}$ is at least three and sometimes reaches to several ten, which means the strong suppression due to the $\gamma$ and $Z$ contributions.

Since the $\gamma$ - and $Z$-exchange cross sections are well-known, we can define the other 
asymmetry $\mathcal{A}^{\prime \prime}$ with extracting the $\gamma$ and $Z$ contributions:

$$
\mathcal{A}^{\prime \prime}=\frac{\sigma(P, P, B, F)-\sigma(P, P, F, B)-\sigma_{\gamma, Z}^{d i f f}}{\sigma(P, P, B, F)+\sigma(P, P, F, B)-\sigma_{\gamma, Z}^{t o t}},
$$

where $\sigma_{\gamma, Z}^{\text {diff }}$ and $\sigma_{\gamma, Z}^{t o t}$ are the $\gamma$ and $Z$ contributions in $\sigma^{\text {diff }}$ and $\sigma^{t o t}$, respectively. Here $\sigma_{\gamma, Z}^{\text {diff }}$ is vanishing. Since we have extracted the background effects of $\gamma$ - and $Z$-exchange from $\mathcal{A}^{\prime}$, the $\mathcal{A}^{\prime \prime}$ is proportional to $\mathcal{A}$ :

$$
\mathcal{A}^{\prime \prime}=\frac{4}{\left(\frac{1}{r}+r\right)\left(\frac{1}{P}+P\right)} \mathcal{A}
$$

When $\mathcal{A}^{\prime \prime}>0.1 \mathcal{A}$ is required, $r>0.3$ for $P=0.1$ [4], and $P>0.13$ for $r=0.2$. The values for $P=0.6$ and $r=0.2$ are shown in Table 3 .

Neglecting the systematic errors and assuming 100\% efficiency, we found that required luminosity to establish the non-zero asymmetry $\mathcal{A}^{\prime \prime}$ is least at moderate value of $\tan \beta$. Only $\gtrsim 20 \mathrm{pb}^{-1}$ of the integreted luminosity enables us to perceive a non-vanishing asymmetry within $1-\sigma$ statistical error for $\tan \beta=15$, and $30 \mathrm{pb}^{-1}$ for $\tan \beta=7$. For $\tan \beta=3$ and $30,0.25 \mathrm{fb}^{-1}$ and $1.5 \mathrm{fb}^{-1}$ are required, respectively.

\section{Conclusions}

We have discussed the interference effect on the cross section of the process $\mu^{+} \mu^{-} \rightarrow$ $t \bar{t}$ with $H$ and $A$ resonances almost degenerated. The interference between $H$ and $A$ bosons arises if muon beams are longitudinally polarized and if we observe the helicities of the top quarks. The interference effect can be measured by observing the difference between the cross sections with appropriate helicity combinations. It has been shown that the existence of the difference can be reliable evidence of the existence of both $H$ and $A$ bosons, while the absence indicates the existence of only $H$ bosons or $A$ bosons around the resonances. It is especially important that the existence of the $H$ and $A$ bosons can be established even if their masses are degenerated, by observing the interference effect in the overlapped resonances. We have estimated an asymmetry between the cross sections 
adopting the MSSM as an example. Even after taking into account the background $\gamma$ - and $Z$-exchange contributions because of incompleteness of the polarizations of muon beams and the measurement of top quark helicities, the absolute values of the asymmetry can be detectable in certain ranges of $\sqrt{s}$. If we can accumulate the luminosities which enable us to perceive the asymmetry, it is possible to learn not only the existence of $H$ and $A$ bosons but also the sign of the product of coupling constants of Higgs bosons to fermions from the sign of the asymmetry.

Acknowledgments The authors thank K. Hagiwara and N. Oshimo for valuable discussions and reading manuscript. They also would like to thank K. Fujii, K. Ikematsu and T. Ohgaki for useful comments. This work is supported in part by the Grant-in-Aid for Scientific Research (No. 11640262) and the Grant-in-Aid for Scientific Research on Priority Areas (No. 11127205) from the Ministry of Education, Science and Culture, Japan. 


\section{References}

[1] V. Barger, M.S. Berger, J.F. Gunion and T. Han, Phys. Rep. 286, 1 (1997).

[2] V. Barger, M.S. Berger, J.F. Gunion and T. Han, Phys. Rev. Lett. 75, 1462 (1995);

B. Kamal, W.J. Marciano and Z. Parsa, in Workshop on Physics at the First Muon Collider and Front End of a Muon Collider, edited by S. Geer and R. Raja, AIP Conference Proceedings 435 p.657 (hep-ph/9712270).

[3] B. Norum and R. Rossmanith, Nucl. Phys. (Proc. Suppl.) B51a, 191 (1996).

[4] C.M. Ankenbrandt et al., Phys. Rev. ST Accel. Beams 2, 081001 (1999);

V. Barger, M.S. Berger, J.F. Gunion and T. Han, Phys. Rev. Lett. 78, 3991 (1997);

J.F. Gunion, in Proceedings of the 5th International Conference on Physics Beyond the Standard Model, Balholm, Norway, 199\%, edited by G. Eigen, P. Osland and B. Stugu (AIP, Woodbury, New York, 1997), p.234 (hep-ph/9707379);

V. Barger, T. Han, and C.-G. Zhou, hep-ph/0002042.

[5] A. Pilaftsis, Phys. Rev. Lett. 77, 4996 (1996);

K.S. Babu, C. Kolda, J. March-Russell and F. Wilczek, Phys. Rev. D59, 016004 (1999);

S.Y. Choi and M. Drees, Phys. Rev. Lett. 81, 5509 (1998);

S.Y. Choi and J.S. Lee, hep-ph/9909315.

[6] B. Grzadkowski, J.F. Gunion and J. Pliszka, hep-ph/0003091.

[7] D. Atwood and A. Soni, Phys. Rev. D52, 6271 (1995).

[8] E. Asakawa, J. Kamoshita, A. Sugamoto and I. Watanabe, hep-ph/9912373, to be published in Eur. Phys. J. C.

[9] A. Djouadi, J. Kalinowski and M. Spira, Comm. Phys. Commun. Res. 108, 56 (1998).

[10] K. Hagiwara, H. Murayama and I. Watanabe, Nucl. Phys. B367, 257 (1991).

[11] M. Jeżabek and J.H. Kühn, Nucl. Phys. B320, 20 (1989);

G. Mahlon and S. Parke, Phys. Rev. D53, 4886 (1996). 
[12] G.A. Ladinsky and C.-P. Yuan, Phys. Rev. D49, 4415 (1994);

R. Frey, in Proceedings of the Workshop on Physics and Experiments with Linear Colliders (LCWS95), Iwate, Japan, Sept., 1995, eds. A. Miyamoto et. al., (World Scientific, 1996), p.144 (hep-ph/9606201);

R. Frey et al., hep-ph/9704243, Report of the Top Physics working group at the 1996 DPF/DPB Snowmass workshop. 


\section{Tables}

Table 1: The helicity dependence of the Higgs amplitudes of $\mu^{+} \mu^{-} \rightarrow H / A \rightarrow t \bar{t}$. We denote $\mathcal{M}_{H / A}^{R R R}$ as $\mathcal{M}_{H / A}$ for simplicity.

\begin{tabular}{|c|r|r|}
\hline & $t_{L} \bar{t}_{L}$ & $t_{R} \bar{t}_{R}$ \\
\hline \multirow{3}{*}{$\mu_{L}^{-} \mu_{L}^{+}$} & $\mathcal{M}_{H}$ & $-\mathcal{M}_{H}$ \\
& $\mathcal{M}_{A}$ & $\mathcal{M}_{A}$ \\
\hline \multirow{3}{*}{$\mu_{R}^{-} \mu_{R}^{+}$} & $-\mathcal{M}_{H}$ & $\mathcal{M}_{H}$ \\
& $\mathcal{M}_{A}$ & $\mathcal{M}_{A}$ \\
\hline
\end{tabular}


Table 2: The masses, the total decay widths, the $\mu^{+} \mu^{-}$decay branching ratios and the $t \bar{t}$ decay branching ratios of the $H$ and $A$ bosons in the MSSM adopted in our numerical simulations.

\begin{tabular}{|c||c|c|c|c|}
\hline $\tan \beta$ & $\begin{array}{c}m_{H} \\
(\mathrm{GeV})\end{array}$ & $\begin{array}{c}\Gamma_{H} \\
(\mathrm{GeV})\end{array}$ & $\begin{array}{c}B r\left(H \rightarrow \mu^{-} \mu^{+}\right) \\
10^{-4}\end{array}$ & $\begin{array}{c}B r(H \rightarrow t \bar{t}) \\
10^{-2}\end{array}$ \\
\hline 3.0 & 403.78 & 0.79 & 0.33 & 74.2 \\
7.0 & 400.71 & 0.50 & 2.84 & 20.7 \\
15.0 & 399.97 & 1.70 & 3.88 & 1.30 \\
30.0 & 399.49 & 6.67 & 3.95 & 0.078 \\
\hline
\end{tabular}

\begin{tabular}{|c||c|c|c|c|}
\hline \hline $\tan \beta$ & $\begin{array}{c}m_{A} \\
(\mathrm{GeV})\end{array}$ & $\begin{array}{c}\Gamma_{A} \\
(\mathrm{GeV})\end{array}$ & $\begin{array}{c}\operatorname{Br}\left(A \rightarrow \mu^{-} \mu^{+}\right) \\
10^{-4}\end{array}$ & $\begin{array}{c}\operatorname{Br}(A \rightarrow t \bar{t}) \\
10^{-2}\end{array}$ \\
\hline 3.0 & 400.00 & 1.75 & 0.15 & 94.6 \\
7.0 & 400.00 & 0.67 & 2.13 & 45.2 \\
15.0 & 400.00 & 1.74 & 3.80 & 3.83 \\
30.0 & 400.00 & 6.69 & 3.95 & 0.25 \\
\hline
\end{tabular}

\section{Figures}

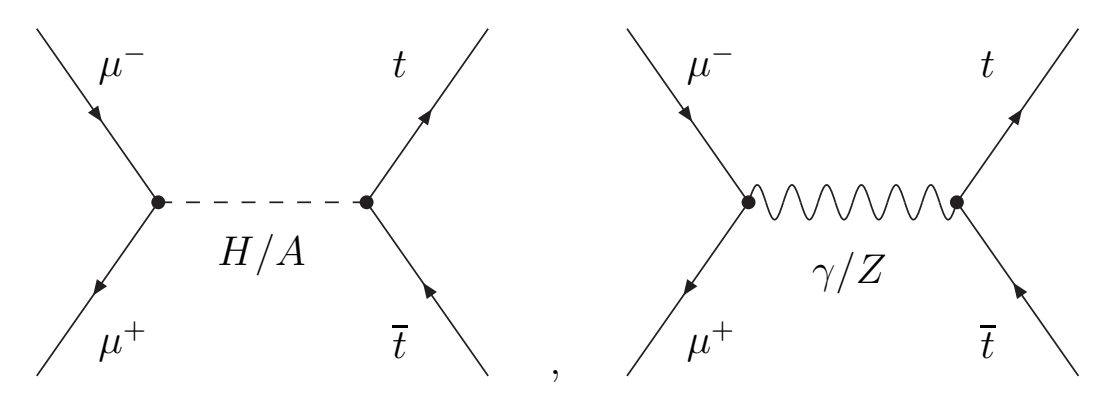

Figure 1: The diagrams of the process $\mu^{+} \mu^{-} \rightarrow t \bar{t}$ around the mass poles of $H$ and $A$ bosons. 
Table 3: The cross sections and the asymmetries. We denote $\sigma_{H, A}(P, P, c, \bar{c})$ with $\gamma$ and $Z$ contributions extracted to be $\sigma_{H, A}(c, \bar{c})$, for simplicity, where $P$ and $r$ are assumed to be +0.6 and 0.2 , respectively, and $c$ and $\bar{c}$ are $F$ (forward) or $B$ (backward) of the angle cut.

\begin{tabular}{|c||c|r|r|r||r||r|r|r|}
\hline $\tan \beta$ & $\begin{array}{c}\sqrt{s} \\
(\mathrm{GeV})\end{array}$ & $\begin{array}{r}\sigma^{L L L L} \\
(\mathrm{fb})\end{array}$ & $\begin{array}{r}\sigma^{L L R R} \\
(\mathrm{fb})\end{array}$ & \multicolumn{1}{|c||}{$\mathcal{A}$} & $\mathcal{A}^{\prime}$ & $\sigma_{H, A}(B, F)$ & $\sigma_{H, A}(F, B)$ & $\mathcal{A}^{\prime \prime}$ \\
& & & $(\mathrm{fb})$ & $(\mathrm{fb})$ & \\
\hline \hline 3.0 & 400.0 & 484.9 & 510.4 & -0.026 & -0.006 & 347.5 & 353.5 & -0.009 \\
& 402.5 & 213.8 & 23.66 & 0.801 & 0.079 & 107.4 & 61.47 & 0.272 \\
& 405.0 & 24.56 & 161.5 & -0.736 & -0.061 & 48.29 & 80.69 & -0.251 \\
& 406.0 & 3.260 & 70.60 & -0.912 & -0.035 & 17.49 & 33.31 & -0.311 \\
\hline 7.0 & 399.0 & 152.2 & 591.2 & -0.591 & -0.113 & 209.1 & 315.4 & -0.203 \\
& 400.0 & 2969.1 & 4071.7 & -0.157 & -0.049 & 2347.1 & 2610.4 & -0.053 \\
& 400.5 & 2358.1 & 1905.0 & 0.106 & 0.032 & 1557.6 & 1447.3 & 0.037 \\
& 401.5 & 1.860 & 684.7 & -0.995 & -0.183 & 159.0 & 321.1 & -0.338 \\
\hline 15.0 & 398.0 & 16.19 & 184.6 & -0.839 & -0.074 & 50.74 & 91.60 & -0.287 \\
& 400.0 & 95.22 & 1226.1 & -0.856 & -0.202 & 329.8 & 599.9 & -0.291 \\
& 401.0 & 42.70 & 527.7 & -0.850 & -0.143 & 142.5 & 257.4 & -0.287 \\
\hline 30.0 & 390.0 & 0.88 & 6.39 & -0.758 & -0.003 & 1.93 & 3.37 & -0.272 \\
& 400.0 & 8.20 & 77.76 & -0.809 & -0.036 & 21.88 & 38.49 & -0.275 \\
& 405.0 & 2.43 & 25.80 & -0.828 & -0.013 & 7.08 & 12.44 & -0.275 \\
\hline
\end{tabular}



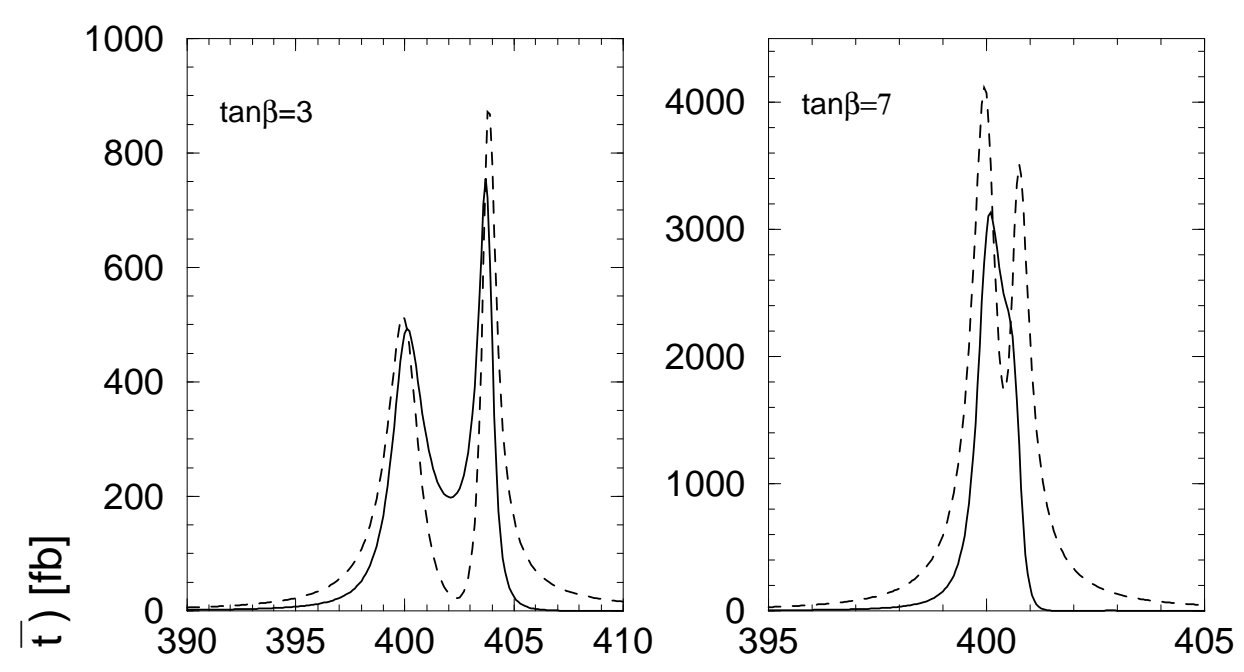

$\underset{\uparrow}{\Psi}$
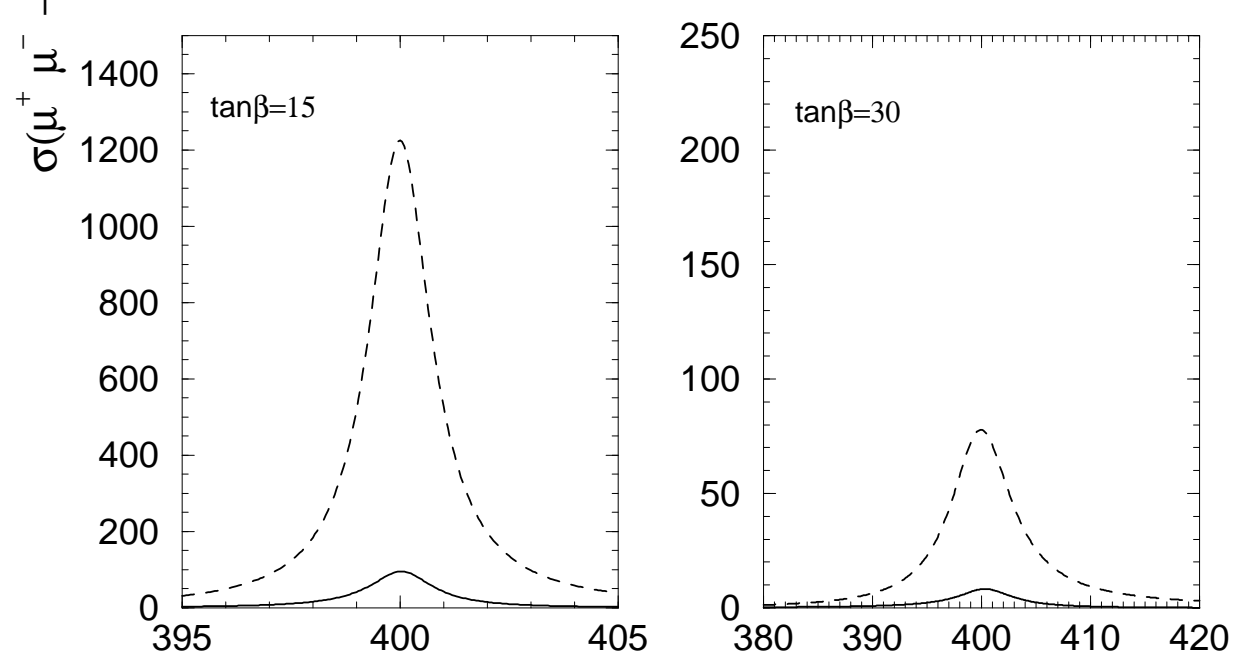

$\sqrt{ } \mathrm{s}[\mathrm{GeV}]$

Figure 2: The cross sections of $\mu^{+} \mu^{-} \rightarrow t \bar{t}$ with Higgs resonances for $\tan \beta=3,7,10$ and 30. The solid curves show the cross sections for $\mu_{L}^{+} \mu_{L}^{-} \rightarrow t_{L} \bar{t}_{L}$ or $\mu_{R}^{+} \mu_{R}^{-} \rightarrow t_{R} \bar{t}_{R}$, the dashed curves for $\mu_{L}^{+} \mu_{L}^{-} \rightarrow t_{R} \bar{t}_{R}$ or $\mu_{R}^{+} \mu_{R}^{-} \rightarrow t_{L} \bar{t}_{L}$. 


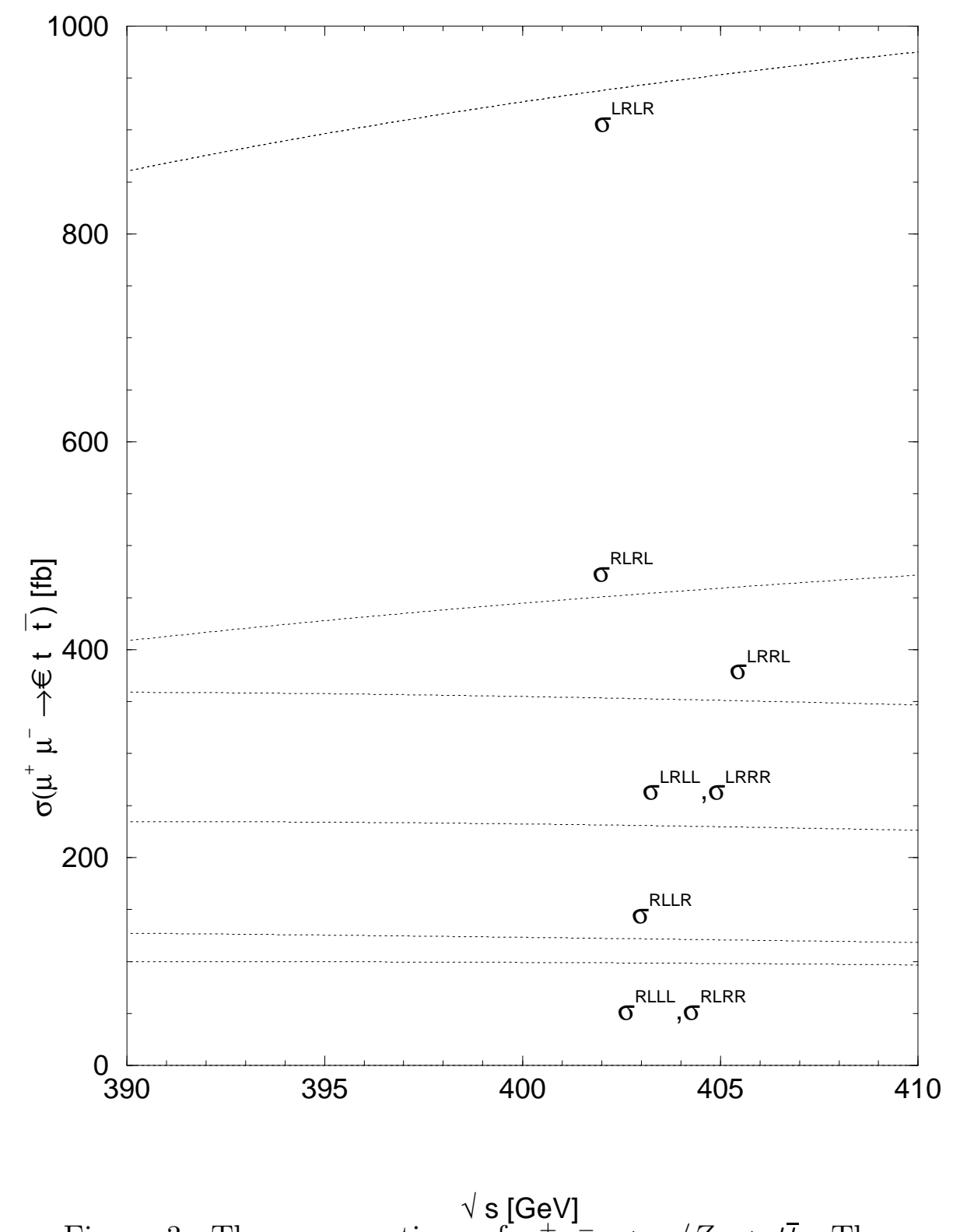

Figure 3: The cross sections of $\mu^{+} \mu^{-} \rightarrow \gamma / Z \rightarrow t \bar{t}$. The cross sections, $\sigma^{L L L L}, \sigma^{L L R R}$, $\sigma^{R R R R}, \sigma^{R R L L}, \sigma^{L L L R}, \sigma^{L L R L}, \sigma^{R R L R}$ and $\sigma^{R R R L}$ are smaller than $0.01[\mathrm{fb}]$. 


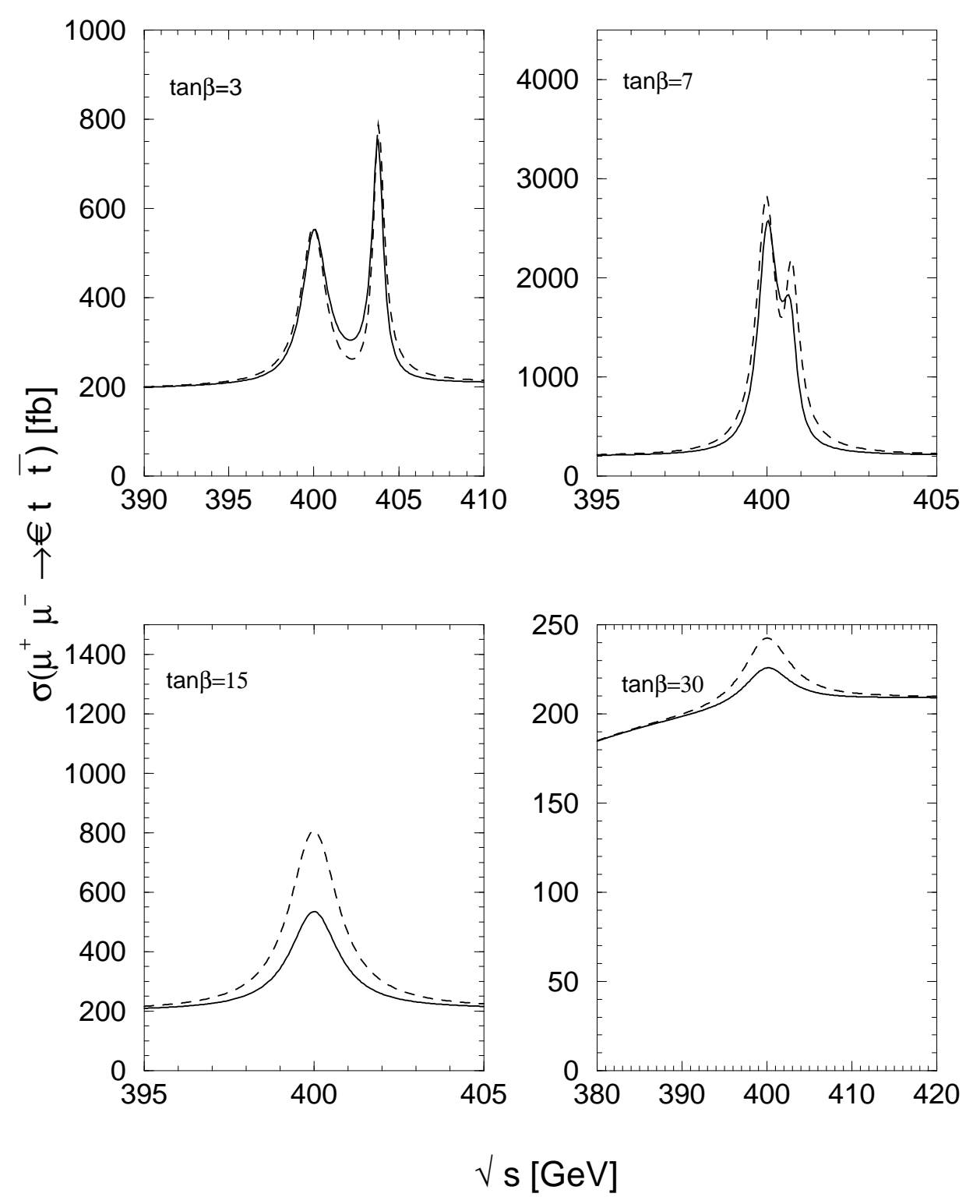

Figure 4: The effective cross sections of $\mu^{+} \mu^{-} \rightarrow t \bar{t}$ for $\tan \beta=3,7,10$ and 30. The solid curves are for $\sigma(P, P, B, F)$ or $\sigma(-P,-P, F, B)$, while the dashed curves for $\sigma(P, P, F, B)$ or $\sigma(-P,-P, B, F)$, with $P=+0.6$ and $r=0.2$. 